\title{
Are We Really Going to Have an Eschatological Future?
}

\author{
Vadim M. Rozin \\ Doctor of Philosophical Sciences, Professor, Institute of Philosophy RAS \\ (Moscow, Russia) \\ E-mail: rozinvm@gmail.com \\ ORCID: 0000-0002-4025-2734
}

The article analyzes the interpretation of the future, in which the person either does not have a place or is expected by a sad fate. Other past religious interpretations of the future are also being considered. The problem arose only in the culture of the new time, when faith and reason were contrasted and understood as opposing realities, and the universe was interpreted in the spirit of natural sciences, that is, as a physical reality. There were various paradoxes. A vivid example of such paradoxes the author sees in the works of Vadim Kazyutinsky, who studied the concepts of the universe and galaxies. In particular, his research allows us to assert that cosmology by basic parameters should be attributed to a scientific discipline of the humanitarian type, which does not exclude the presence in it of various physical and other natural sciences disciplines. The position of the well-known Russian philosopher Alexey Losev, who points to the boundaries of explanation in the spirit of the natural sciences, is also analyzed. In the second half of the article, alternative scenarios (Vadim Kazyutinsky, Hannah Arendt and the author) are discussed to overcome the pessimistic understanding of the future. For the author it is humanitarian nature of philosophy. For Kazyutinsky, the worldview that the Stoics professed, as well as faith man and humanity. For Hannah Arendt - the clarification of the crisis of our thought and civilization. The conclusion is drawn that the considered vision of reality, characteristic of rationalism and natural sciences, is an alternative, and not one.

Keywords: future, man, universe, galaxies, scenarios, alternative

Received: February 5, 2018; accepted: May 3, 2018

Philosophy and Cosmology, Volume 21, 2018: 32-41

DOI: $10.29202 /$ phil-cosm/21/4

Recently I watched on TV a series of amazing films about origin of planets, galaxies and the evolution of the Universe. The films bewitched, it seemed to me that I was on a building site or in the theatre of "creation of the Universe" and lived through the conception, formation and death of space worlds; but at the same time something discomposed me, though I could not understand what exactly. Then I realised: the filmmakers, relying on modern astronomical researches, depictured the future where either there was no place for humans at all, or a sad fate awaited them. The mankind may perish at any time for various reasons: the Earth may collide with another celestial body (a comet, a huge asteroid), or lose son magnetic field

(C) Rozin, Vadim, 2018 
due to an internal magma movement, or this field may be destroyed by a flash of a supernova (moreover, the authors of the program claim that one of the nearest stars will blow up just in a little while, will become a supernova, and immense flows of its radiation, according to the estimates of the astronomers, will rush off directly to our Earth). There are also other scenarios of the Earth and mankind death as a result of natural evolution of the Universe, for example, metamorphoses at dispersal of galaxies or, on the contrary, their collapse in a superdense and extremely small kernel; and all this is not an imagination, but rigorous calculations based on astronomical observations and laws of the nature. There is no reason to disbelieve them, they should be trusted - and as a result an alarm and so-called eschatological expectations appear.

Of course, it is not for the first time that the mankind expects a doomsday, for example, at the turn of the $15^{\text {th }}$ century Russia prepared for the doomsday, and everything confirmed the approach of this terrible event: calculations of the Church, natural signs (a comet passed, an earthquake occurred, the country was captured by a drought), terrible events mentioned in the Apocalypse (wars, civil strifes, people running wild); but the doomsday still does not come, though each next time eschatological expectations do not become less sharp and more usual.

It is the same situation nowadays. Modern people, trusting natural scientists, have a presentiment of a close end of the world, and do not see an alternative: after all what can be done if, for example, the magnetic field of the Earth disappears or a giant asteroid crashes against our planet. However, eschatological expectations amplify and even are provoked by fantasies of a human himself: movies and books about off-worlders, terrible technogenic accidents, world wars, etc. became very popular. There is a feeling that people strike fear in themselves. Is not it because scientists deprived them involuntarily of the future?

However, why do we trust scientists so much? Because they — together with engineers continuously certify technogenic civilization. In fact, the reality of culture needs to be certified (new generations come, conditions and customs change, but the culture is alive so long as its reality is maintained and renewed). For instance, we certify the belief in existence of the nature and its laws that forms an essential factor of our technogenic civilization by creating machines and other technical structures that operate efficiently, thereby confirming the laws of the nature disclosed by the man.

In the very first, archaic culture, souls and spirits (demons) were part of reality and have to be certified too. This still may be seen in Tibet during a magic "chod" (derived from a verb meaning "to cut off, to destroy") ceremony. This ceremony is based on belief in existence of demons. "Tibet, - writes David Neal, — is the country of demons", which, according to beliefs and legends, are more numerous than the population of the country; they hunt for people and animals, stealing from them the "breath of life" that demons consume as food. "The functions of official Lamaism include subordination of demons, their re-education, converting them into obedient servants, and in case they do not obey - their neutralization or destruction" [David-Neel, 1992: 99]. Probably, one of objectives of the "chod" ritual is to train young monks ("trapa") communicating with demons, but in an extremely peculiar form, putting themselves at the mercy of demons. In doing so, the victim, addressing to demons, cries out the following spells:

"Throughout a boundless number of centuries, in the course of repeating existences, I borrowed from uncountable beings on account of their lives my food, my clothes and various benefits to contain my body in good health, in pleasure and to protect it from death. Now I am paying the debts, offering for destruction my body which I loved and cherished so. I give my flesh to the thirsty and blood eager ones, my skin to those who are nude; I throw my bones 
into the fire for those who suffer from cold. I give my happiness to unfortunate and my breath of life to those dying." In doing so, the trapa imagines that his will in an image of a female deity comes out through the top of the head, in one stroke cuts off its head, then hands and feet with a hatchet, skins and disembowels it. Demons fly together from all directions for this "entertainment" and, champing savourily, suck flowing blood, tear meat, and crack bones. "The performance, — writes David-Nil, — is intended to intimidate the performer, and is so skilful that during the ceremony some trapas suddenly go crazy and even fall dead." 2

In the culture of ancient kingdoms (Ancient Egypt, Babylon, and early antiquity), the belief in gods was confirmed by means of numerous sacral practices. They included regular offices in temples devoted to various gods, mystery plays like Orphic-Dionysian ones and the life of esoteric communities, for example, Pythagorean ones. It should be taken into account that during these practices people not only met gods or tried on their clothes, or prepared themselves for divine life, but each time were convinced of existence of respective reality. I think my thought is clear, and there is no need to give more examples.

So, why a modern person should not trust astronomers who study planets, galaxies and the Universe using the most sophisticated equipment (telescopes, satellites, various devices) created on the basis of laws of the nature? Besides, they reveal characteristics of space within the framework of these laws, using mathematics, computers, and natural sciences. In this, context scientists (astronomers) persuade us of such future where mankind may expect at any time a cataclysm and the Doomsday. It seems that there is no alternative to such future. However, let us not hurry and consider some arguments in favour of such an alternative. We may call the first argument cultural and historical.

It is known that for many millennia people thought that the Universe is not a physical world, but rather either gods (the sun, the moon, the stars - all these were gods) or, as in the Middle Ages, a world created for a human by the God. In fact, the Universe was turned towards the man. For example, the sun understood by Sumerian as god, warmed the Earth, lit, and gave life. From the point of view of an antique or medieval person, the Universe, the life on the Earth and the reason were conceived simultaneously as they had to serve each other. Platon's Demiurg through his omnibenevolence, almost love to humans, conceives and creates Space and at the same time, the man. Aristotle's Reason — a live conceiving and reflexing deity coinciding with the sky and the space moves planets by the power of its thought. A person thinks correctly, believes the Stagirite, if it imitates the Reason. The God of the Middle Ages created a man in his own image; and, having created the world, intended it for the man. "The fire by its nature, wrote John Chrysostom, - strives upwards, rushes and flies skywards..., but with the Sun the God made absolutely opposite: he turned its beams to the Earth and made the light fall down, as if speaking to it: look down and shine people: you have been created for them."3

The problem arose only during modern times when the belief and the reason were brought apart, the Universe was interpreted in the spirit of natural sciences, which is as a physical reality, and it turned out, as Pascal said, that a human is a miserable reed, and its place in the Universe is unclear and insignificant. From the point of view of such Universe, there is no sense of existence of a human. At the suggestion of Francis Bacon and Galilee, the nature began to be understood as "constrained by art" (in experiment and in mechanism). A man turned everything that he could reach in the nature into equipment. Today - in the same vein - he wants to master the Solar system.

\footnotetext{
${ }^{1}$ Ibid. Pages 105-106.

${ }^{2}$ Ibid. Page 100.

${ }^{3}$ Writings of John Chrysostom, Archbishop Constantinople. SPb., 1896, Vol. 2, Book 1, page 394.
} 
Yet, he cannot reach the Universe. It seems so easy: to build spaceships, to fly and to study all corners of the Universe; but after all, it is only our intentions, pure speculations. Today and in a relatively long term people will not fly anywhere, they even do not know whether it is practicable in principle 4 . Besides, there is one more important difficulty that is the second argument: our knowledge of the Universe changes all the time. In the ancient world, the Universe was understood as the world of gods; in the Middle Ages, this pagan understanding was rejected; in modern times, people scarified also the Christian understanding of the world. Since the second half of the $19^{\text {th }}$ century, our ideas of the Universe change almost every 50 years. Given current development of science and equipment, our ideas of the Universe are likely to change drastically more than once in the centuries to come.

However, knowledge methods and knowledge itself change also. Currently, many physicists agree that a humanitarian approach is not contraindicated to them. A humanitarian discriminates and studies his/her object from the point of view of his/her own interest, his/her research unfolds, according to Gadamer, within a hermeneutic circle; and there are as much circles as traditions and significant approaches. Then maybe the Universe is an object of human sciences? In other words, I begin discussion of the third argument, from knowledge and methodology.

In fact, a long-term research of Vadim Kazyutinsky who has always believed that the Universe is a physical object, showed absolutely other things. ${ }^{5}$ It showed that at the level of metagalaxies and the Universe it is not possible to carry out the main procedures of certification of physical reality. One cannot set up a decisive experiment. It is impossible to stop at one true interpretation of the observed phenomena; on the contrary, in the field of the Universe studies many different, quite reasonable theories and concepts have been developed and compete on equal terms. ${ }^{6}$ An "anthropic principle" (Brandon Kartor) has been introduced into cosmology according to which "phenomena that we expect to observe, have to be limited by conditions necessary for our existence as observers."

${ }^{4}$ Cf. "Of course, - Hannah Arendt notices, - our current plans do not go beyond investigation of the immediately surrounding part of the Universe, that infinitesimal area that the mankind is able to reach, even moving with velocity of light. Given the duration of a human life - the only absolute restriction which remained at the moment - it is very improbable that we will ever reach much further" [Arendt, 2014: 403].

5 “According to Abram Zelmanov's definition, - Vadim Kazyutinsky writes, - cosmology is "a physical doctrine about the Universe as a whole, including the theory of the whole world covered by astronomical observations as a part of the Universe". Kazyutinsky esteems in the same way, saying that "despite singularity of the object of cosmology it nevertheless represents a physical object" (Vadim Kazyutinsky. Traditions and revolution in modern astronomy. (Doctoral dissertation). Institute of philosophy of the Russian Academy of Sciences. M, 1999. Pages 10, 32).

6 "The problem in this case, - Hannah Arendt writes, - is that apparently almost any axiom allows a coordinated justification, and to such an extent that there is an impression that people may prove eventually any hypothesis that they want to be accepted, and this applies not only to purely cogitative structures like comprehensive interpretations of the history supported well with facts but also to natural sciences" [Arendt, 2014: 131-132].

7 "Observed properties, - Kazyutinsky explains, — are rigidly connected with numerical values of some fundamental physical constants. If these constants were just a little different, the existence of atoms, stars, galaxies in the Universe would be impossible, same as the emergence of conditions which led to the appearance of the man, the observer" (Ibid. Page 49). Herefrom, one theological explanation: "the man is the purpose of evolution of the Universe defined by transcendental conditions" (Ibid. Page 50). However, if the Universe defines the sense and the purpose of existence of the human, then a cosmologist, studying it, thereby cognizes himself and human nature (a spirit cognizes the spiritual?). Aren't we here in the territory of human sciences? 
Polemizing with Joseph Shklovsky claiming in 1979 that though "A lot of things are not clear yet and has to be learned, but the "master plan", the interrelation of objects, and, above all — the history development (of the Universe) - are understood and passed into category of absolute truth", Kazyutinsky writes the following. "Doubts are generated by three circumstances. First, modern evolutionary theories in astrophysics have not yet a sufficient number of predictions confirmed on their basis, i.e. is fail to fully satisfy to the criterion of substantiality adopted in the science. On the contrary, some predictions do not come true (for example, the flow of solar neutrinos turned out to be much less than the one predicted by the theory). Secondly, despite almost semicentennial researches of active processes in galaxies, many of them have no quite a reliable explanation yet. In fact, we do not know still what happens in kernels of galaxies. Thirdly, numerous and very sophisticated attempts to resolve "paradox of weight" were not crowned with success so far. According to the contemporary view, accumulations of galaxies have to be stationary; but for this purpose, it is necessary to assume that $95-98 \%$ of the mass of the Universe substance are in an invisible state ("hidden mass, dark matter"). All attempts to understand the physical nature of hidden masses failed so far. ${ }^{8}$ Well, this is some kind of a natural science where only $2-5 \%$ are explained and the main mechanisms are not clear!

The "singularity issue" was especially intolerant: in compliance with it, moving back to the beginning of dispersal of galaxies, we shall come to the zero point where many physical parameters (the mass of substance, radiuses of particles and others) gain infinite or zero values, losing thereby a physical meaning. Then such a fundamental question arises as "What was "prior" to singularity?" Some researchers "carefully express their opinion in the sense that currently, there is no reasonable physical answer to this question." ${ }^{\prime 9}$ If it is not present in physical reality, perhaps, this problem can be solved within the framework of human sciences? How else answers given by many cosmologists may be interpreted: this question is senseless because already Augustine stated, "the time had to appear together with the Universe."10

To sum up, in cosmology everything is approximately the same as in human sciences. Analyzing Kazyutinsky's works, I even formulated a paradox: within our Solar system, we deal with physical reality since, nevertheless, an experiment and real technical practice is possible (say, have the rocket reached Mars or Saturn or not?), but outside the Solar system, especially in the Universe, we may speak only about humanitarian reality.

Cosmology in all respects has to be referred to a scientific discipline of humanitarian type that does not exclude availability therein of various physical and other natural-science disciplines. The object of cosmology (similar to the objects of biology, cultural science, sociology) cannot be described by one scientific discipline. The "cosmological reality" is multilevel with specific objective laws acting on each level, which laws have to be described by various cosmological theories. From the point of view of science philosophy, the Universe as an object of studying of cosmology, represents ideal objects of humanitarian theories which are created based on facts (astronomical observations and their interpretation), in the process of implementation of cosmologists' values and approach, carrying out a humanitarian discourse (for example, treatment of astronomical observations as peculiar texts and activity of Space), taking into consideration humanitarian nature of the Universe (plurality of cosmological theories, anthropic principle, etc.).

\footnotetext{
${ }^{8}$ Ibid. Pages 26, 27, 30, 31.

${ }^{9}$ Ibid. Page 33.

${ }^{10}$ Ibid. Page 34.
} 
Then again, if the Universe studies are referred to human sciences, then first, forecasts of astrophysicists about the death of our Earth - it not an exact calculation, but a hypothetical knowledge reflecting rather current state of cosmology than reality, and secondly, as a humanitarian knowledge it is also incorrect as it works not for the benefit of humans. Orientation towards a human is one of particularities of human sciences: humanitarian knowledge as Michael Bakhtin noted - not only helps to understand cultural texts, but also constitutes reality-creating conditions (meanings and vision) for a human life.

Now, the fourth argument. Usually natural sciences make it possible to gain knowledge providing for forecasting, calculation and management of natural phenomena thereby enabling us to create modern sophisticated machines. That is, eventually, natural sciences work for the benefit of people, enabling them to master the nature. However, there are also challenges here: engineering based on natural sciences, initiates processes which were not conceived and calculated and objectively are often negative for a man. For example, mastering nuclear energy, on the one hand, of course, is a source of many benefits, but on the other, it is a threat to the existence of the mankind (given that we still do not know what to do with nuclear waste which constantly increases, and we have not managed yet to create nuclear power plants that would be reliable enough to exclude probability of nuclear disasters).

Incidentally, studying of the Universe gives absolutely another picture. Something, in fact, becomes clear and can be calculated (for example how one stars die, but from the matter ejected as a result of this process new planets, stars and galaxies gradually are born). Though in general, studying the Universe, on the one hand, generates full misunderstanding of the events (why at first all matter was concentrated in a midget volume, why there was the "Big Bang", why the dispersal of galaxies accelerates, etc.), and on the other hand, it leaves no room to the future (not to the future of stars and planets, but to the future of the humankind).

Pondering over this circumstance, Kazyutinsky in conversation with the author said that there is a certain contradiction between the grandness of the Universe, and processes that occur there (their scale and character blow the mind) and the role of the man in the Universe. Let us take, for example, he said, the dispersal of galaxies or huge emissions, discharges, absorption of matter and energy observed in supernovas, black holes or quasars. And let us compare them with short duration and negligibility of human life. How all this may be reconciled? Can it be true that we shall disappear forever if any asteroid collides with the Earth, or we shall gradually die out during the next implosion of galaxies?

However, already Isaac Newton answered such doubts. When its theory did not match perfectly the observed reality, he told: "And what is the God for? He is putting everything in order". We may find a similar discourse with Alexey Losev, explaining to Vladimir Bibikhin, why he is a believer. Losev said, "It struck me. I lived my life, could not, and cannot understand. I know for sure - I cannot understand. Eventually, everything comes to the question of the good and the evil. The God is the creator; he is all-powerful - and what happens here? Cannot he eliminate all this disgrace with one movement of his little finger? However, yes, he can. Then why he does not want to? It is a secret... Angels have fallen. The devil, a fallen angel - accepts everything, except absolute existence of the God. Cannot God prevent him from doing evil? Ha-ha! And why God does not do it? A secret.

Anyway, the believer is the one who managed to see clearly this secret. The others say that there is no God; but this is rationalism, and tomfoolery... While the belief begins when the God is crucified. The GOD is crucified!!! When you try to understand this, you see: it is a secret. Of course both ancient and new ones new this secret. Aristotle naively in one place of "Metaphysics" says one thing and in another place, a different thing. The ideas seem to be 
correct in both places; but if you had asked him: how so, in one place you have an absolute mind, the prime mover which manages everything, and in the other - some gobbledygook, he would have run away as a bug from a finger; and if he were a believer, he would tell: it is a secret. Therefore I did not want to make an absolute of "Metaphysics". They also know the evil! So why does not it come to their mind to ask what is happening: the eternal prime mover on the one hand, and such a disgrace on the other hand? How so? If they had asked this, question and had answered: it is a secret, and then they would have become believers.

Some say: make the sign of the Cross and your illness will pass. Nonsense! On the contrary, the one who crosses himself risks to face a bigger evil. This association of the good and the evil is inexplicable - an honest, good person suddenly finds himself in an unpleasant situation, in a stalemate. He may be even killed, a noble man, the best of all. So what does the God think? A secret. This is a secret; and when a person comprehended this secret, he/ she does not need any cross or other symbols... Of course, maybe there are some sacraments, which alleviate the situation; but what if after a chrismation a person got sick and died? I will not be surprised - it means that it had to be this way. It means that such was the God's will.

Therefore, without belief there is nothing but vulgar optimism and rationalism. Of course, often you pray, recompose yourself and the illness passes, and if on the contrary, the illness aggravates and the person dies - then thy will be done! This is an inconceivable and inexplicable mystery!

Therefore, stating the tiresome, boring metaphysics claiming absoluteness (a strong, divine peace arrangement) - I think that the relativity is right there. The sky, of course, moves for ages. This god, at least the lower one, moves through the whole movement of the heavenly arch. - However, if just for one second it turns out that this arch is not there, if for some one moment all this heavenly arch drops out, blows up, breaks, disappeared - I will not be surprised. Because I am a believer; and if I were a pagan - then yes, of course, I would tell that we have chaos here on the Earth, through tranquil stars move constantly, eternally, steadily, etc. From the Christian point of view, this is relative, but paganism is an absolutization of the whole world. Well, let Platon and Aristotle believe that this arrangement is indestructible - let them trust. Although if suddenly there is an accident, they would not know what to do - but I will say: the God's secret came true; let it be."11

Vadim Kazyutinsky, who for many years have been dealing with the "contact issue", in fact, also adopted the viewpoint of belief, however not religious one, but that of "stoical belief" in selectness and uniqueness of the mankind. All observations, he told me in a personal conversation, show that we are alone in the Universe; and it should be recognized that it is the most adequate - I would even say courageous - position. Yes, the life and the reason are unique and, we are alone in the Universe. Of course, science fiction writers write all the time about other planets and forms of life; but they simply do not understand what life is, and do not know researches of astrophysicists. It is really amazing how the Earth was adapted for emergence of life. It is located neither too close to the Sun and nor too far from it. In the first case, it would be too hot on the Earth, as on Venus, therefore life would be impossible, and in the second case - eternal cold and ice as on Pluto, and certainly, protein-based life would never appear here. It turns out that it is some kind of preset harmony, or, more exactly, a priori orientation towards life. Our sun is neither too young nor too old; in both opposite cases, life would be impossible. Our planet is surrounded with an atmosphere and a magnetic field that reliably protect the life from ultraviolet light and pernicious space beams. We are located

${ }^{11}$ Vladimir Bibikhin. From Alexey Losev's stories. The Siberian orthodox newspaper. 2005. No.1. http://www.ihtus.ru/012005/c1.shtml 
in an ideally quiet place of the galaxy. The more I think of this, Kazyutinsky told, the more I m convinced that the Earth is a unique place for emergence of life. There is a feeling that the Universe has been created just for us as though it is a specially designed test tube and a laboratory for life. If I were a believer, Vadim concluded, and then it was exactly on the basis of data of modern astronomy that I would come to a conclusion about divine creation of life on the Earth, and in this context the Universe was conceived by the God as a maternal bosom.

I did not want to adopt such nevertheless religious version; at the same time, I tried to overcome a physicalistic representation of the Universe embraced by Kazyutinsky. However, why we have to think, I retorted, that the Solar system is only a physical object. Maybe, at the same time it is some other organism, say cosmic, vital, and sacral. I do not know. A physical aspect does not deny other aspects. For example, mentality and thinking are not reduced to physiology and brain, but the latter are one of prerequisites of the former. It would be strange to deny availability of mentality or to reduce it to the brain on the basis of the fact that the brain is a substrate of thinking and other mental processes. By the way, the brain is only one of substrates of mentality, but the other are not less essential — signs, activity, communication; and it is the same in the Universe: there are both physical and nonphysical aspects. Rather, the question is what we shall consider as basic at solution of certain tasks. For instance if I want to understand the place of the man in the Universe so as to inspire him. What in that case I have to take as a basis of the thought - physical or nonphysical reality?

Kazyutinsky reflected and after a while answered, "It seems, I devised one scenario. Once a human came out of the nature. Now he changes it. However, inaptly so far, often to the detriment of himself; but I am sure that he will learn, and will change the nature, taking into consideration that he is its organic element. Yet sooner or later, let it be through one thousand years though, seemingly, much earlier, the man will completely change the appearance and the structure of the Earth. Arguing the same way, it is possible to assume the mankind will not stop there: it will start "customising" the Solar system at first, then the Galaxy, and at last there will be the turn of the Universe. The result of these titanic efforts that will stretch over millions and billions of years will be transformation of the Universe into a living being combining the nature and the mankind at the same time. Of course, I understand that you will accuse me right there of arrogance: not only that the man destroys the nature on the Earth, but I aim the Universe already. Nevertheless I solved the task set by you: I created a scenario of the Universe - meaningful and optimistic for the human".

I agreed but noticed that, perhaps, I would try to build another scenario. It is difficult to assume, I told, that on such a long way (millions and billions of years) the mankind will keep its purpose, that it will not change drastically and that the issue of "customisation" of the Universe will still interest it. Besides, I have already paid your attention to the fact that our idea of the Universe will change more than one time. I would also add that, most probably, we would not be able to understand the Universe nature for a long time. That is we see something, some formation, maybe organic, but not live, maybe this is some form of life, or perhaps something else that does not come to our mind simply due to our backwardness.

In this case, it is more reasonable to act carefully, following the principle of establishment of "contact", somewhat in the way as characters of "Solaris" acted; but here the case is much more difficult. With Stanislaw Lem it was at least clear where is the whole and where are the borders: the planet and Ocean-Solaris thereon. Although in case with the Universe, everything is unclear: where is the whole, whether it is the only or not, where are the borders, what do they represent, etc. All this shall be borne in mind and we shall act accordingly: try to listen to the Universe in all possible ways, address it, tell about ourselves, be ready to any 
answer, the most unexpected one, study and think all the time, trying not to lose sight of all uncertainty and limitation of our knowledge of the Universe. Perhaps, this scenario is not the most optimistic, of course yours, Vadim, is more inspiring, but the mine, agree, is more realistic and reasonable from the point of view of the knowledge and opportunities available to us. Such was our conversation somewhere at the very end of the $90^{\text {ies }}$.

The same year, as though on purpose, the book "Between the Past and the Future" was published, where Hannah Arend as early as in the $60^{\text {ies }}$ of the last century discussed in fact our subject. In particular, she demonstrated that such a strange representation of the Universe were instilled to us by scientists (physics and astrophysics) who began to take somewhat detached view of the man and the Earth - from space, and with the use of tools ${ }^{12}$ based on mathematics, replacing sensory perception and traditional categories (reason, law, etc.) by a new reality. "Data, Arendt writes, investigated by modern physics, flash as "mysterious envoys from the real world" ... Addington without slightest doubts assumes that these physical data arise from "the real world", i.e. by default more real than the world in which we live ...

It is known how strongly Einstein was reluctant to sacrifice the principle of causality that was demanded by the Planck quantum theory; his main objection, certainly, was that if it carries on like this, the Universe will lose any regularity as though God managed the world by "playing dice." 13

Arendt also analysed the position of scientists who, for the sake of beauty and search of truth, i.e. esoteric thoughts, rather than for the sake of public benefit, insist on a new picture of the world. However, Arendt shows that the result of these titanic esoteric searches was paradoxical and sad for scientists themselves: in the world opened by them not beauty and harmony, but accidentally, chaos and nonsense reigned. "According to Schrödinger, the new Universe that we try to "subdue", it is not just "inaccessible in practice, but even inconceivable" because "try as we may, our thought is wrong; perhaps it is not so senseless as a "triangular circle', but much more senseless than a 'winged lion.",

Least of all scientists wanted to "conquer space", to fly to the Moon or something like that ... Actually nothing else than the search of "true reality" forced them to lose faith in phenomena, in phenomena in the aspect in which they are disclosed to human feelings and reason. They were inspired by an extreme love to harmony and regularity which learned them that if they wanted to open comprehensive beauty and the order of the whole (Universe), they to go beyond any given sequence or series of events. This may explain why they seem not so much to be tormented with the fact that their discoveries served the invention of the most deadly devices, as worry because of the crash of all the most cherished ideals of regularity and need. These ideals were lost when scientists found out that there were no any indivisible matter, any a-tomos, that we live in an extending, unlimited Universe and that randomness, seemingly, predominates everywhere where there is a "true reality"; the physical world completely goes beyond the area accessible to human feelings or any devices which leveled down their gawkiness."14

At last, Arendt concludes that, moving in the said direction, the man meet only himself ("the things that are made by the man and is his next mask"), but such succession of events threatens with death to the man. "The situation as it appears today, oddly resembles a careful-

12 "The modern science started from thoughts inconceivable earlier (Copernicus imagined himself "standing on the Sun ... examining planets") and from things unprecedented before (the Galilee's telescope pierced the space separating the Earth from the sky, and "with all reliability of sensory perception" presented to the human knowledge secrets of stars)" [Arend, 2014: 401-402].

${ }^{13}$ Hannah Arendt. Cit. Writings, pages 392, 398.

${ }^{14}$ Ibid. Pages 395, 400-401. 
ly thought over confirmation of the remark made by Franz Kafka at the dawn of these changes: the man, he told, "found an Archimedean point, but used it against himself; probably, only on these conditions he was allowed to find it." After all the conquest of space, the search of a point beyond the Earth using which the planet could be moved, as if "unhinged", — it not an accidental result of science. From the very beginning it was not a science "about the nature", but a science about the Universe: not physics, but astrophysics ... The conquest of space and the science which made it possible brought us too close to this point. If they once really reach it, the status of the man will not only become lower by all criteria known to us, but will cease to exist"15. Let us recapitulate what was said.

How it is possible to understand reflections of Losev, Kazyutinsky, Hannah Arendt and my own? The point is that there is an alternative, and not the only one, to the vision of reality set by rationalism and natural science. For Losev it is a peculiar belief. For the author it is a humanitarian philosophy. For Kazyutinsky it is a stoical mental outlook, belief in the man and the Mankind. For Hannah Arendt it is an awareness of the crisis of our thought and our civilization. "Christians, she writes, considered as immortal only a certain person and anything else in this world: neither mankind in general, nor even Earth ... The modern times opened potential immortality of the mankind ... The history, stretching infinitely both in the past and in the future, it is capable to provide immortality on Earth almost as a Greek polis or the Roman Republic provided to human life and acts - to the extent those revealed or created something essential and great - a specific human and terrestrial imperishability in this world." "Speaking about earthly history, we live within a process which does not have neither the beginning, nor the end and thereby not allowing us to cherish eschatological expectations." 17

Yet, Arendt notices, when it became clear that "all processes on the Earth and in the Universe are either man-made, or potentially man-made" 18 , it became impossible to comprehend this process giving a meaning to the life of a new European man and a hope for immortality. Nevertheless, Arendt believes - probably following the ideas of her teacher, Martin Heidegger - that if the man thinks over his critically activity, including his own thinking, and realises that "it is the Earth, but not the Universe, which is the house and the center of dwelling of mortal people" $" 19$, in this case, there is a hope.

However, the alternatives are not limited to these views.

\section{[D] References}

Arendt, Hannah. Conquest of space and the man's status. Between the past and the future. Moscow, 2014. 416 p.

Bibikhin, Vladimir. From Alexey Losev's stories. The Siberian orthodox newspaper. 1, 2005. http://www.ihtus.ru/012005/c1.shtml

David-Neel, Alexandra. With Mystics and Magicians in Tibet. Moscow, 1992. 232 p.

Kazyutinsky, Vadim. Traditions and revolution in modern astronomy. (Doctoral dissertation). Institute of philosophy of the Russian Academy of Sciences. Moscow, 1999.

Writings of John Chrysostom, Archbishop Constantinople. SPb., 1896, Vol. 2, Book 1, 491 p.

\footnotetext{
15 Ibid. Pages 409, 412.

${ }^{16}$ Ibid. Pages 112, 115.

${ }^{17}$ Ibid. Page 104.

${ }^{18}$ Ibid. Page 136.

${ }^{19}$ Ibid. Page 411.
} 